\title{
Terminal Myelocystocele : Pathoembryogenesis and Clinical Features
}

\author{
Ji Yeoun Lee, ${ }^{1,2}$ Kyung Hyun Kim, ${ }^{1}$ Kyu-Chang Wang ${ }^{1}$ \\ Division of Pediatric Neurosurgery,' Seoul National University Children's Hospital, Seoul, Korea \\ Department of Anatomy and Cell Biology, ${ }^{2}$ Seoul National University College of Medicine, Seoul, Korea
}

There has been confusion in the classification of terminal myelocystocele (TMCC) due to its diverse morphology and vague pathoembryogenesis. TMCC could be summarized as having the essential features of an elongated caudal spinal cord extruding out of the dorsal extraspinal space that fuses with the subcutaneous fat, which is in the shape of a trumpet-shaped cerebrospinal fluidfilled cyst. The extraspinal portion of the extruded spinal cord is nonfunctional. The morphological features suggest that TMCC is formed during secondary neurulation, specifically the failure of the degeneration of the secondary neural tube near the time of the terminal balloon. This review discusses the definition, as well as the clinical and surgical features, of TMCC with special emphasis on its pathoembryogenesis.

Key Words : Neurulation · Congenital anomalies · Nervous system.

\section{INTRODUCTION}

Congenital anomalies are formed due to the occurrence of an error during the dynamic process of development. Therefore, these anomalies generally exhibit a wide variety in morphology. It is not uncommon to find two separate entities with similar morphology or the same entity with quite different appearances. Therefore, the classification of congenital anomalies according to gross morphology may cause confusion. Terminal myelocystocele (TMCC) exhibits elaborately different morphologies, and it is a representative entity for which the controversy about its classification was solved using the underlying pathoembryogenesis.
Although TMCC is not a common type of spinal dysraphism encountered by clinicians, its unique clinical features, operative details and its theory of pathoembryogenesis deserve special attention.

\section{DEFINITION}

Pang et al. .) $^{2}$ summarized and 'demystified' the ponderous characteristics of TMCC into essential and nonessential features. Essential (obligatory) features must be found in the anomaly to be called a TMCC, and nonessential (optional) features account for the high variability in the morphology of

- Received : March 4, 2020 •Revised : March 17, 2020 •Accepted : March 31, 2020

- Address for reprints : Ji Yeoun Lee

Department of Anatomy and Cell Biology, Seoul National University College of Medicine; Division of Pediatric Neurosurgery, Seoul National University Children's Hospital, 101 Daehak-ro, Jongno-gu, Seoul 03080, Korea

Tel : +82-2-740-8205, Fax : +82-2-749-3485, E-mail : femiddang@naver.com, ORCID : https://orcid.org/0000-0003-0464-7605

This is an Open Access article distributed under the terms of the Creative Commons Attribution Non-Commercial License (http://creativecommons.org/licenses/by-nc/4.0) which permits unrestricted non-commercial use, distribution, and reproduction in any medium, provided the original work is properly cited. 
the entity ${ }^{1)}$.

The caudal spinal cord is elongated in all cases of TMCC, and its terminal end exits the spinal canal for attachment to the subcutaneous fat of intact skin. The end of the spinal cord itself is protruded, and it flares out in the shape of a trumpet, in which some cerebrospinal fluid (CSF)-filled cysts are found. The CSF-filled space may or may not be directly connected to the hydromyelic canal of the more proximal spinal cord (Fig. 1).

The subarachnoid space surrounding the terminal end of the spinal cord also protrudes out of the spinal canal. The amount of the subarachnoid space varies from slightly wider than normal to a huge cavern and may extend extraspinally to surround the neck of the trumpet (Fig. 2). Hydromyelia in the more proximal, intraspinal portion of the spinal cord may vary greatly. The thickness of the subcutaneous fat layer that the trumpet is attached to also varies between patients. The amount of the subarachnoid space and the subcutaneous fat cause the wide variety in the size of the sacral hump. The sacrum may also have an anomalous morphology that ranges from hypoplastic to supernumerary with a straight to backward-pointing curvature.

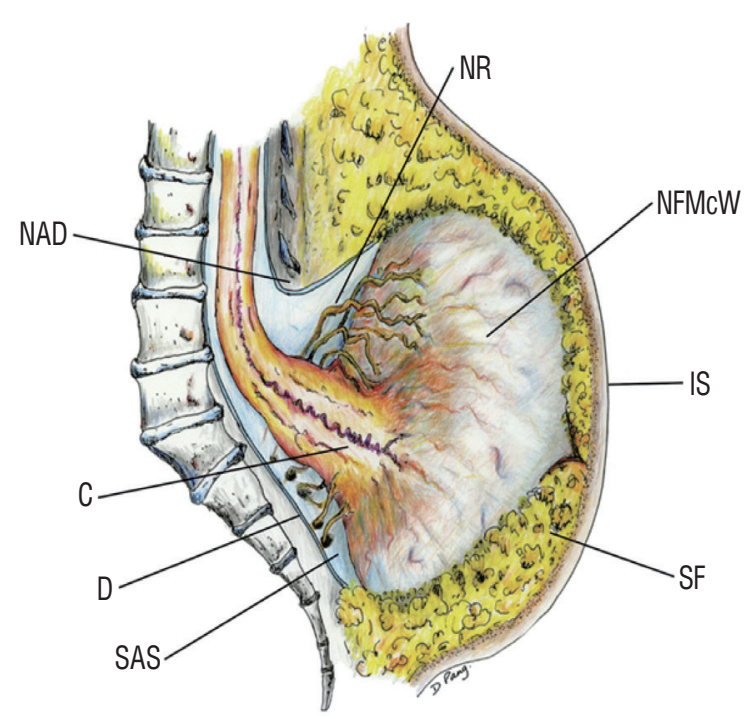

Fig. 1. A schematic drawing showing the essential features of terminal myelocystocele. The caudal end of the spinal cord is extended out of the spinal canal and attached to the subcutaneous fat in the shape of a flared trumpet. Adapted from Pang et al. ${ }^{6}$ with permission from Oxford University Press. NR : functional sacral roots, NAD : neural arch defect, NFMcW : nonfunctional myelocystocele wall, C : conus, IS : intact skin, D : dura, SAS : subarachnoid space, SF : subcutaneous fat.

\section{EMBRYOLOGY}

McLone and Naidich ${ }^{5)}$ postulated a short theory on the pathoembryology of TMCC as the remnant of a dilated terminal ventricle caused by the CSF that failed to exit from the early neural tube before canalization. The attempt to explain the important phenotype of the anomaly was noteworthy, but the academic speculation was too brief for any detailed explanation of the normal embryology and its erroneous result.

Based on an earlier study on the morphology of secondary neurulation in chick embryos ${ }^{9)}$, Pang et al. ${ }^{6)}$ noticed a striking resemblance between TMCC and a point during secondary neurulation in which the distal end of the secondary neural tube dilated into a balloon-like structure (terminal balloon, Fig. 3). The balloon initially starts as a mere widening of the cavity that is continuous with the central canal. Because the degeneration of the secondary neurulation progresses with the continued thinning of the secondary neural tube, the terminal balloon dilates somewhat, and the gap between the balloon and cutaneous ectoderm decreases, almost to a point where the wall of the balloon is fused to the skin. The dilated balloon and proximal cord resemble the morphology of the 'trumpet' attached to the subcutaneous layer, which is an essential fea-
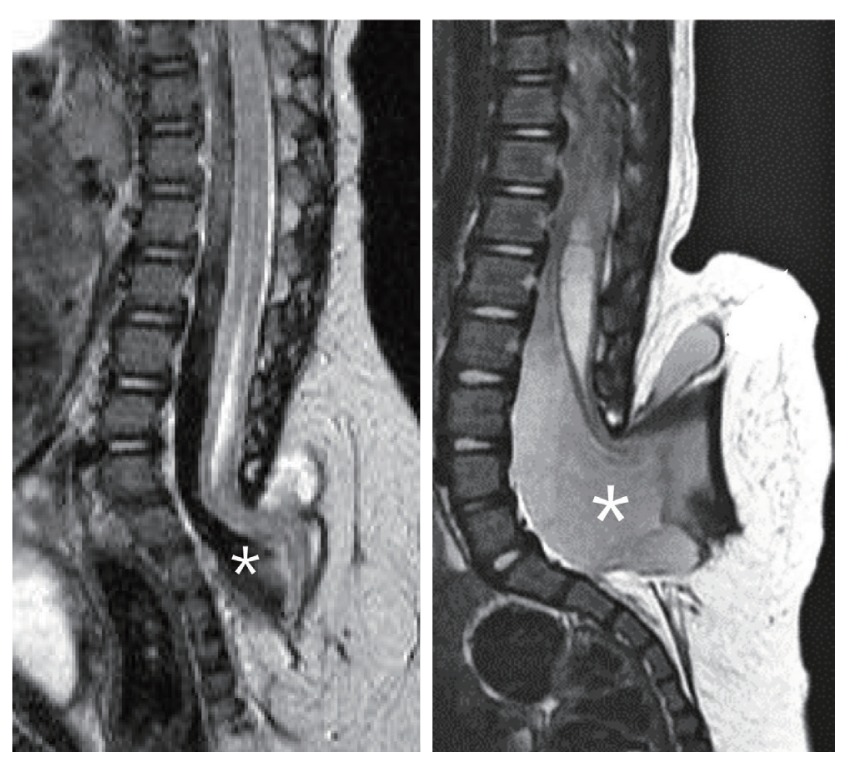

Fig. 2. Two cases of terminal myelocystocele showing a minimal amount of subarachnoid space (left) and a very wide extraspinal extension of the subarachnoid space (right). Asterisk $\left(^{*}\right)$ denotes the subarachnoid space in both pictures. Adapted from Pang et al. ${ }^{6}$ with permission from Oxford University Press. 
ture of TMCC. The terminal balloon eventually shrinks in normal development as the degeneration of the secondary neural tube progresses further. The gap between the caudal end of the cord and the cutaneous ectoderm increases dramatically (Fig. 4). However, the arrest of degeneration at the moment of terminal balloon may result in the formation of TMCC. This hypothesis was further supported in a later study in which a structure similar to the terminal balloon was found in human fetal tissue ${ }^{8)}$.

Details of normal development gave further clues about the

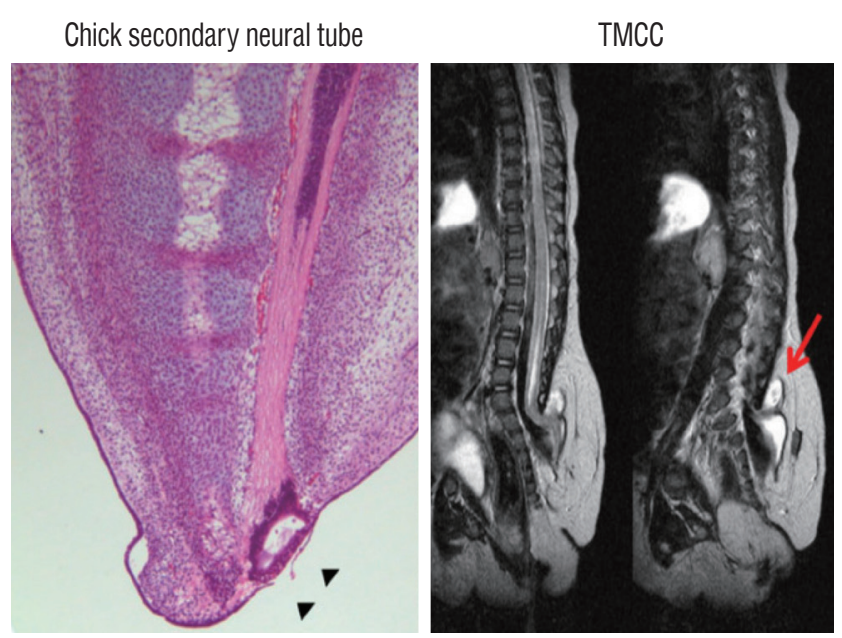

Fig. 3. The striking resemblance between chick secondary neurulation (left) and the sagittal MRI images of a prototypical TMCC patient (right). Note the apposition of the chick terminal balloon to the surface ectoderm (arrowheads), which is comparable to the subcutaneous position of the human TMCC (arrow). Adapted from Lee et al. ${ }^{4)}$ with permission from Springer Nature. MRI : magnetic resonance imaging, TMCC : terminal myelocystocele. nonessential features of TMCC. Because the gap between the balloon and cutaneous ectoderm goes through the steps of decrement and increment, the proximity of the flared end of the trumpet to the skin (i.e., the depth of the subcutaneous fat layer) depends on the time of the arrest. The degree of the hydromyelia may also be different depending on the time sequence of the degeneration of the proximal cord and shrinkage of the balloon. The study showed that the neuroectoderm and cutaneous ectoderm were separate layers, even though they may be just two single layers of cells, and this layering explained how the flared surface of the trumpet is always able to peel off from the subcutaneous fat during surgery ${ }^{6}$.

\section{IMAGING FEATURES}

There is a low-lying cord, and the most terminal end of the cord protrudes through a dorsal defect. The end of the cord is attached to the subcutaneous fat layer with a flaring of the end to form the shape of the horn of the trumpet. The trumpet is thin-walled and fluid-filled, and it forms the "myelocystocele". A thin layer of tissue is generally visible that separates the CSF-filled cyst and the overlying subcutaneous fat layer by 'occluding' the wide-mouthed base of the trumpet.

Significant extension of the subcutaneous fat into the spinal canal is unusual in a typical TMCC. However, the trumpetlike flaring of the distal spinal cord is unusual in typical caudal lumbosacral lipomatous malformation (LLM) with extraspinal extension. Nonetheless, there are examples of a 'gray
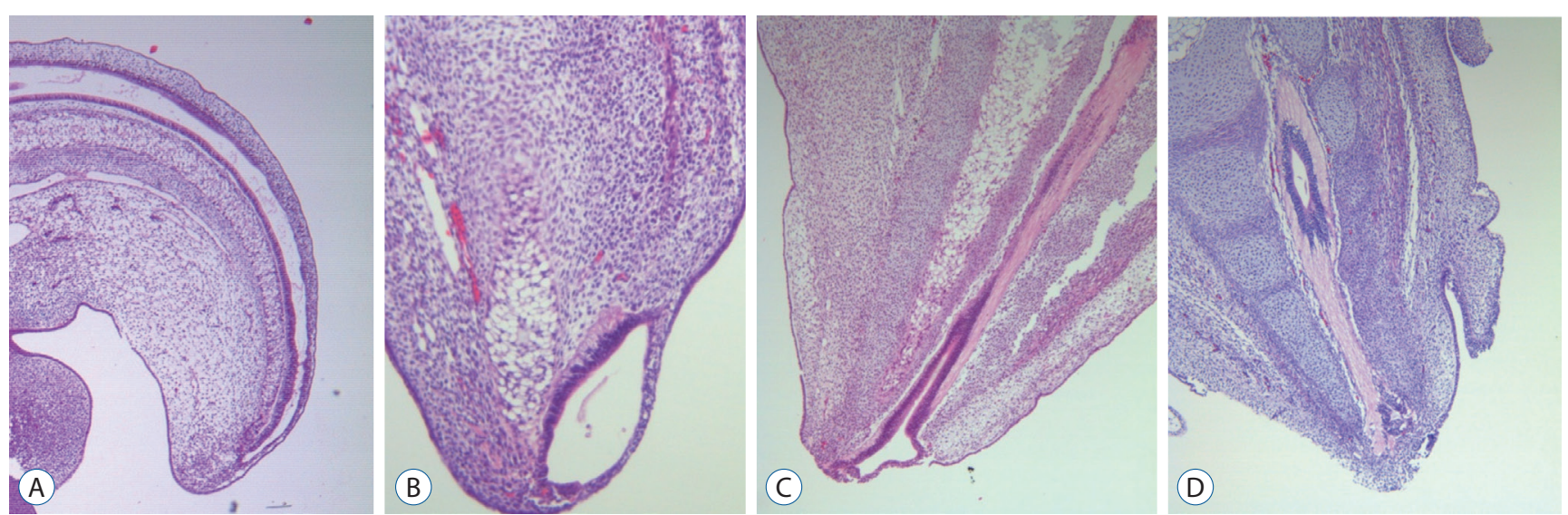

Fig. 4. A series of H\&E images of the developing chick embryos showing the formation and degeneration of the terminal balloon. A : Formation of the cavitary conjoining of primary and secondary neural tubes $(x 40) . B$ : Appearance of the terminal balloon $(\times 100)$. C : Shrinkage of the terminal balloon $(\times 40)$. $\mathrm{D}$ : Degeneration of the terminal balloon and detachment of the medullary cord from the surface ectoderm $(\times 40)$. 
zone' between a TMCC and caudal LLM with extraspinal extension, with some extension of fat into the spinal canal in TMCC or some dilatation of central canal at the distal spinal cord in LLM with extraspinal extension. TMCC and LLM may be 'sister lesions' because the LLM is a connection between the neuroectoderm and surface ectoderm via the expanded subcutaneous fat.

The amount of subarachnoid space, hydromyelia, and subcutaneous adipose layer may vary greatly. Almost no subarachnoid space may be visible or a big sac may be present that widens the posterior bone defect. The width and length (extent of the longitudinal size) of hydromyelia may also vary from normal to extremely high (Fig. 5). The lumbosacral hump may be huge in cases where the subcutaneous fat layer is thick.

\section{SYMPTOMS}

The symptoms of TMCC are similar to LLM. Because the lump on the sacral area is not negligible, the diagnosis is made

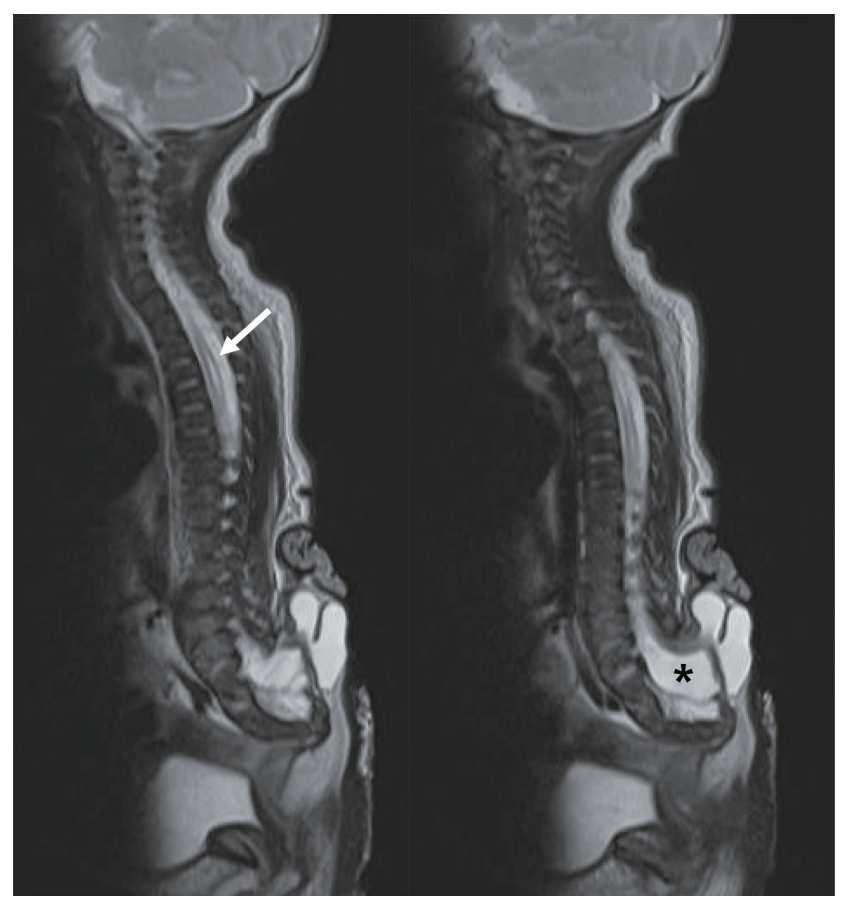

Fig. 5. A series of MRI T2 sagittal images of a 1-week-old girl with terminal myelocystocele. Note the extensively wide subarachnoid space (right, asterisk) and syringomyelia up to the upper thoracic level (left, arrow). MRI : magnetic resonance imaging. soon after birth. Many patients are symptomatic on physical examination and detailed urological or electrophysiological studies at diagnosis, but neurologically normal subjects are encountered. However, Pang et al. ${ }^{6}$ showed that patients diagnosed later in life were all symptomatic with lower extremity motor impairment and neurogenic bladder, suggesting the poor natural history of TMCC. Therefore, it is recommended that surgical management be considered even for asymptomatic patients.

A unique clinical feature to be considered in the management of TMCC is that enlargement of the cyst is a warning sign for the rapid progression of neurological deficits. The progression may be very rapid, such as a foot deformity that develop in less than 2 months. The enlargement of the cyst may directly stretch the neural tissue (spinal cord) in the dorsal direction and cause rapid deterioration. Therefore, parents should be warned about enlargement of the cyst size, and medical advice should be sought immediately in this circumstance ${ }^{4)}$.

\section{OPERATIVE PROCEDURE}

It is important that the normal dura and spinal cord are defined via the bony exposure of at least one level cephalad to the defect. Durotomy is performed to reveal the normal spinal cord, the neck of the trumpet (where it protrudes out of the bony defect), and the flare of the trumpet that is attached to the subcutaneous fat. After a rough layout of the spinal cord proximal and distal to the trumpet neck is visualized, the functioning rootlets should be identified at the caudal end of the normal spinal cord inside the spinal canal. Proper intraoperative electrophysiological monitoring is absolutely necessary for the surgical management of TMCC. Stimulation (0.5-2 $\mathrm{mA}$ ) of the rootlets allows identification of the last functioning rootlets, and the location of the functioning conus may be speculated and further confirmed using direct stimulation (5-10 mA) of the cord.

The wall of the cyst may be opened at the midline after the functional and spatial layout is understood. The smooth lining of the inner wall is exposed, and its direct connection with the hydromyelic cavity may be seen. After opening of the cyst, the surgeon may view the inner and outer aspect of the entire trumpet. Direct stimulation of the cyst wall at a higher inten- 
sity $(5-10 \mathrm{~mA})$ in a distal-to-proximal direction enables the identification of the region where the neural tissue shows no response to electric stimulation, i.e., 'nonfunctioning. Together with the previously identified last functioning sacral rootlets and the functioning conus, the margin for maximal, safe resection may be determined. After excision of the nonfunctioning part of the spinal cord, pial reconstruction of the reduced bulk of the cord should be performed. A generous duroplasty should be done to maximize the cord-to-sac ratio. We use bovine pericardium for duroplasty ${ }^{6}$. Operative photographs are shown in Fig. 6.

Although the syringomyelia may be quite huge in some cases, syringotomy is not necessary. The syringomyelia in cases of cord tethering is almost always resolved by untethering, and direct syringotomy should be avoided.

\section{PATHOLOGY}

The pathological features of the excised 'trumpet' were reported and support the fact that the excised portion is a 'nonfunctioning', 'normally should have been degenerated' spinal $\operatorname{cord}^{6}$. First, despite the implication of the name 'myelocystocele', the smooth inner lining was mostly devoid of ependyma, which were only observed in occasional spots within the core of the tissues populating the deep folds as ciliated ependymal epithelium. Second, the tissue contained neurons and glial cells, but it was nothing like the normal neural tube. Third, numerous neurons were found haphazardly throughout the entire specimen, but without any functional patterns.

\section{ASSOCIATED ANOMALIES}

The association with midline abdominal and pelvic anomalies was noted ${ }^{7}$. The pathoembryogenesis was explained by the fact that the caudal cell mass, which is the main player of secondary neurulation, is responsible for the neural tube of the sacrococcygeal area and the genitourinary, intestinal structures of the region. The most famous type of anomaly is the omphalocele, exstrophy of the bladder, imperforate anus, and spinal abnormalities (OEIS), in which TMCC is the spinal abnormality. The actual frequency of the co-occurrence may not be very high, as suggested in previous reports ${ }^{3}$.

Hydrocephalus is also frequently mentioned as an associated condition. However, the underlying pathoembryogenesis that links the two conditions is not very obvious, unlike the association of hydrocephalus with meningomyelocele. The actual rate of hydrocephalus in TMCC varies widely between reports $^{2,5,6)}$. Nonetheless, it is harmless for clinicians to pay attention to the possibility of hydrocephalus in TMCC patients.
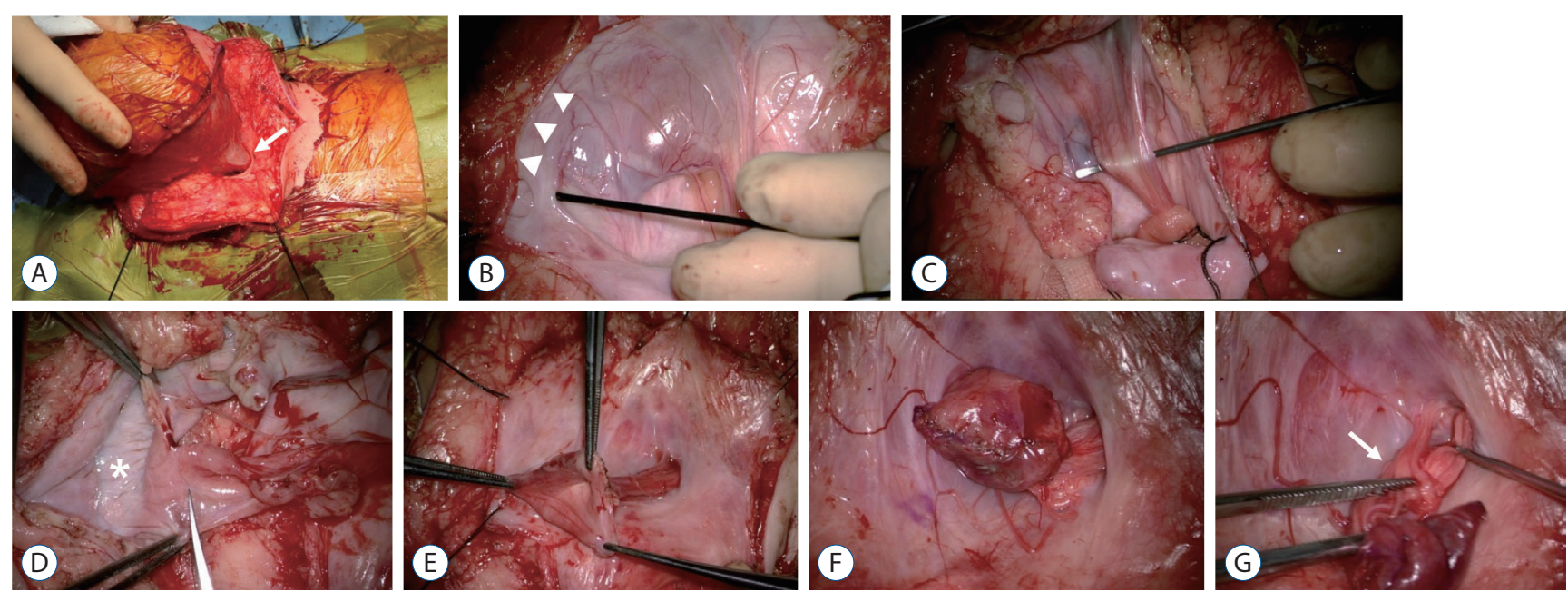

Fig. 6. Surgical sequence in a case with terminal myelocytocele. A : The neck of the trumpet (arrow) is seen extending out of the fascia defect into the huge sac. B : After dural opening, the margin at which the myelocystocele sac joins the subcutaneous fat is shown (arrowheads). C : The rostral spinal cord flares out into the proximal part of the enormous cyst. D: The sac wall is opened to expose the inner lining (asterisk), and a trimming of the margin of the neural placode is performed. Neural placode before (E) and after pial reconstruction (F). G : Nerve roots (arrow) arise from the basal side of the proximal sac and stream back into the spinal canal. 


\section{CONCLUSION}

TMCC is a rare anomaly that needs special attention due to its unique pathoembryogenesis. Clinically, cyst enlargement should be cautiously monitored, and surgical untethering should be performed with meticulous monitoring.

\section{CONFLICTS OF INTEREST}

No potential conflict of interest relevant to this article was reported.

\section{INFORMED CONSENT}

This type of study does not require informed consent.

\section{AUTHOR CONTRIBUTIONS}

Conceptualization : KCW, JYL

Data curation : KKH, JYL

Formal analysis : JYL

Methodology : KKH, JYL

Visualization : $\mathrm{KKH}$

Writing - original draft : JYL, KCW

Writing - review \& editing : KKH, KCW, JYL

\section{ORCID}

Ji Yeoun Lee https://orcid.org/0000-0003-0464-7605

Kyung Hyun Kim https://orcid.org/0000-0002-8238-2043

Kyu-Chang Wang https://orcid.org/0000-0001-7440-6650

\section{- Acknowledgements}

This research was supported by the Bio \& Medical Technology Development Program of the National Research Foundation (NRF) of Korea funded by the Ministry of Science \& ICT (NRF-2018-M3A9H3021707).

\section{References}

1. Barkovich AJ, Kuzniecky RI, Dobyns WB : Radiologic classification of malformations of cortical development. Curr Opin Neurol 14 : 145149, 2001

2. Byrd SE, Harvey C, McLone DG, Darling CF : Imaging of terminal myelocystoceles. J Natl Med Assoc 88 : 510-516, 1996

3. Choi S, McComb JG : Long-term outcome of terminal myelocystocele patients. Pediatr Neurosurg $32: 86-91,2000$

4. Lee JY, Phi JH, Kim SK, Cho BK, Wang KC : Urgent surgery is needed when cyst enlarges in terminal myelocystoceles. Childs Nerv Syst 27 : 2149-2153, 2011

5. McLone DG, Naidich TP : Terminal myelocystocele. Neurosurgery 16 : 36-43, 1985

6. Pang D, Zovickian J, Lee JY, Moes GS, Wang KC : Terminal myelocystocele: surgical observations and theory of embryogenesis. Neurosurgery 70 : 1383-1404; discussion 1404-1405, 2012

7. Tandon V, Garg K, Mahapatra AK : Terminal myelocystocele: a series of 30 cases and review of the literature. Pediatr Neurosurg 48 : 229235, 2012

8. Yang HJ, Lee DH, Lee YJ, Chi JG, Lee JY, Phi JH, et al. : Secondary neurulation of human embryos: morphological changes and the expression of neuronal antigens. Childs Nerv Syst 30 : 73-82, 2014

9. Yang HJ, Wang KC, Chi JG, Lee MS, Lee YJ, Kim SK, et al. : Neural differentiation of caudal cell mass (secondary neurulation) in chick embryos: Hamburger and Hamilton Stages 16-45. Brain Res Dev Brain Res 142 : 31-36, 2003 